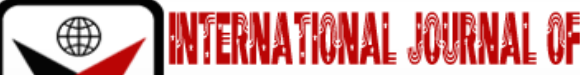

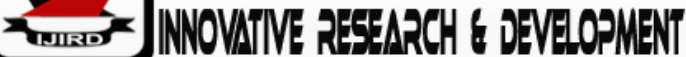

ISSN 2278-0211 (Online)

\section{Bayesian Hierarchical Approach to Modelling Risk of Miscarriage during First Trimester of Subsequent Intrauterine Pregnancy in Women}

\author{
Blasio Omulama Amoche \\ M.Sc. Statistics Student, Department of Mathematics, \\ Kibabii University, Kenya \\ Dr. Joseph Omondi Ouno \\ Senior Lecturer, Department of Mathematics and Physical Sciences, \\ Maasai Mara University, Kenya \\ Barack Otieno Abonyo \\ Professor, Consultant and Research Scientist in Pulmonary Physiology, \\ College of Medicine, Uzima University, Kenya \\ Dr. Boniface Otieno Kwach \\ Senior Lecturer, Department of Mathematics, \\ Kibabii University, Kenya
}

\section{Abstract:}

Background: Accuracy of pregnancy outcome predictions are essential for clinicians to be effective in handling pregnant women experiencing symptoms of miscarriage when presented in the Early Pregnancy Units (EPU). Therefore, the focus of this study is to improve accuracy in modeling risk of miscarriage during first trimester of subsequent intrauterine pregnancy. To achieve this, the study formulates and analyze a Bayesian two-level random intercept logistic model M2 that takes into consideration hierarchical structure in the subsequent pregnancy outcome. This research work is motivated by the recent epidemiological research works that indicated a two-level structure in the subsequent pregnancy outcome with respect to previous pregnancy outcome.

Materials and Methods: The proposed Bayesian hierarchical logistic random intercept model M2 was formulated using the concept of multilevel and Bayesian modeling. Analysis of the proposed Bayesian hierarchical logistic random intercept modelM2 was implemented using the Markov Chain Monte Carlo simulation, in specific Gibbs sampling. Assessment and comparison of the proposed Bayesian hierarchical logistic random intercept model to other reviewed classical approaches using the DIC and AIC.

Results: Assessment of model fitness using DIC and AIC indicates that the proposed Bayesian two-level random intercept logistic modelM2has the lowest value of DIC=121.2 among both classical and Bayesian approaches M0, M1 and M2. This indicates that the proposed Bayesian model M2 is the most plausible approach in modeling risk of miscarriage during first trimester of subsequent intrauterine pregnancy. Assessment of the between cluster variation as a result of the two-level hierarchical structure in subsequent pregnancy outcome, and prior information which, according to this study, are revealed to be essential in modeling risk of miscarriage during first trimester of the subsequent intrauterine pregnancy in women. Relative biases for the model parameter estimates were all below maximum threshold of $\mid 0.2 /$ thus indicating accurate model estimations.

Conclusion: The proposed Bayesian two-level random intercept logistic model M2 is the most plausible approach in modeling risk of miscarriage during first trimester of subsequent pregnancy. Taking into account between previous pregnancy outcome cluster variations and updating observations with prior information is essential to improve accuracy in modeling the risk of miscarriage during first trimester of subsequent pregnancy.

Keywords: Posterior distribution, prior information, parameter, estimation, miscarriage, trimester

\section{Introduction}

In most cases for women, pregnancy is a moment of happiness and excitement when planning for a future. Globally, the proportion of most of the miscarriages that are recognized clinically is $12 \%$ to $15 \%$ of the total number of known pregnancies and in most cases happens during the first trimester of pregnancy [19][17]. Experience of pregnancy loss, however, takes away the pride of an expectant woman, causing distress both physiologically and psychologically, and can also lead to unplanned high economic costs. Therefore, accurate prediction of pregnancy outcomes deems essential for 
clinicians to be effective in handling pregnant women experiencing symptoms of miscarriage when presented in the Early Pregnancy Units (EPU). Reviewed clinical studies shows that the chances of reoccurrence of a miscarriage are high in cases of a miscarriage in previous pregnancy outcome, and reoccurrence of stillbirth is high for women who have experienced a previous stillbirth [2][9]. This indicates a hierarchical structure in subsequent pregnancy outcome in women. However, previous approaches used to model risk of miscarriage in subsequent pregnancy [7][10][14] fail to recognize hierarchical structure in the pregnancy outcome data assuming that units of analysis are independent observations. This therefore results into inaccurate predictions and estimations due to underestimation of standard errors hence overestimation of statistical significance, especially for the higher-level predictor variables [4]. The reviewed studies have also failed to incorporate prior beliefs in modeling subsequent pregnancy outcome during first trimester. Therefore, this study proposes a Bayesian two-level random intercept logistic model for estimating risk of miscarriage during first trimester of subsequent intrauterine pregnancy in women. Our proposed model takes into account the between cluster variations that arise from the previous pregnancy outcome clustering, and also update observed data with relevant prior information. The model aims at improving accuracy in modeling risk of miscarriage during first trimester of the subsequent pregnancy. Analysis of the proposed Bayesian model was implemented using the Markov Chain Monte Carlo (MCMC) simulation, in specific Gibbs sampler which was used to sample parameter estimates from the model posterior distribution. The study focused on women with subsequent intrauterine pregnancy outcomes in Kenya. Secondary data from the Jaramogi Oginga Odinga Teaching and Referral Hospital (JOOTRH) in Kenya which composed of 115 women with both previous and subsequent pregnancy outcome cases of the year 2019 was used in assessment and analysis of our proposed model.

\section{Model Formulation and Analysis}

Previous epidemiological study [6] adopted use of a single level model, say M0 where the model only considers individual women with subsequent pregnancy outcome in modeling the risk of miscarriage and fails to consider the hierarchical structure in subsequent pregnancy. Let $j=1, \ldots, \mathrm{J}$ be the possible previous pregnancy outcome where $\mathrm{J}$ is the number of possible clusters of previous pregnancy outcomes. In this study, we take $\mathrm{J}=3$ which represents three possibilities of outcomes (that is, Miscarriage, Still birth and live birth). Suppose $i=1, \ldots, n_{j}$ is an individual woman involved in the study where $n_{j}$ is the number of women in a $j^{\text {th }}$ previous pregnancy outcome cluster. Let $y_{i j}$ be a binary subsequent pregnancy outcome for the $i^{\text {th }}$ woman who experienced $j^{\text {th }}$ previous pregnancy outcome such that $y_{i j}=1$ when subsequent pregnancy outcome turns out to be a miscarriage, and $y_{i j}=0$ otherwise. Then individual subsequent pregnancy outcome is distributed as a Bernoulli with a probability of $\theta_{i j}$ such that;

$$
y_{i j} \sim \operatorname{Ber}\left(\theta_{i j}\right) \text {, for all } i \in\left(1,2, \ldots, n_{j}\right), j \in(1,2, \ldots, J)
$$

\subsection{Two-Level Empty Model (M1)}

At this stage of model formulation, the study formulates model M1 that involves level-2 previous pregnancy outcome cluster random effects but exclusive of miscarriage determinants for subsequent pregnancy. In equation (2.1), $\theta_{i j}$ is modeled with regard to a logit link function $g($.$) as a latent variable such that;$

$$
g\left(\theta_{j}\right)=\eta=\beta_{0}+u_{0 j}
$$

where $\eta$ is a linear predictor such that $\eta=\operatorname{logit}\left(\theta_{j}\right)$ and $\theta_{j}$ is the probability of experiencing a miscarriage during first trimester of subsequent pregnancy for woman with $j^{\text {th }}$ previous pregnancy outcome. $\beta_{0}$ is the overall population mean logodds of miscarriage in a subsequent pregnancy outcome, and $u_{0 j} ; j \in(1,2, \ldots, \mathrm{J})$ is the previous pregnancy outcome cluster random effects for which in this study it is assumed that $u_{0 j} \sim N\left(0, \sigma_{u 0}^{2}\right)$.

\subsection{Two-Level Random Intercept Model (M2)}

The study further improves on the two-level empty model M1by introducing explanatory variables into the model. Suppose $X_{h i j}$ is a $h^{\text {th }}$ risk factor associated with miscarriage for $i^{\text {th }}$ woman in the $j^{\text {th }}$ previous pregnancy outcome cluster and $h=1,2, \ldots, k$ number of risk factors. Extension of the empty model M1in equation (2.2) by adding $k$ risk factors associated to miscarriage results to a logistic random effect model M2as follows;

$$
\operatorname{logit}\left(\theta_{i j}\right)=\eta=\beta_{0}+\sum_{h=1}^{k} \beta_{h} X_{h i j}+u_{0 j}
$$

where $u_{0 j}$ forms the random part of the model, that is, random previous pregnancy outcome cluster effect and that $u_{0 j} \sim N\left(0, \sigma_{u 0}^{2}\right)$. The fixed part of the model in equation (2.3) is formed by $\beta_{0}+\sum_{h=1}^{k} \beta_{h} X_{h i j}$ since the regression coefficients $\boldsymbol{\beta}=\beta_{0}, \beta_{1}, \ldots, \beta_{k}$ are fixed [43], and therefore, probability of a miscarriage $\left(y_{i j}=1\right)$ is given as;

$$
\theta_{j}=\frac{\exp \left(\beta_{0}+\sum_{h=1}^{k} \beta_{h} X_{h i j}+u_{0 j}\right)}{1+\exp \left(\beta_{0}+\sum_{h=1}^{k} \beta_{h} X_{h i j}+u_{0 j}\right)}
$$

\subsection{Bayesian Two-Level Random Intercept Model}

At this stage of model formulation, the study incorporates prior information which may include clinician judgement, in the modeling of the chances of miscarriage for subsequent pregnancy with respect to the $j^{\text {th }}$ cluster of previous pregnancy outcome. To achieve this, the study first determined the likelihood function of the two-level model in equation (2.3), then updated the likelihood with prior information to obtain a posterior distribution of unknown parameters of interest $\beta_{0}$ and $\sigma_{u 0}^{2}$ in the model. 


\subsubsection{Likelihood of the Two-Level Random Intercept Model}

Likelihood is a measure of goodness of fit for a given statistical model to data sample for designated values of unknown parameters [35]. Sample joint probability distribution is used to form the likelihood, however, the likelihood is used and viewed as a function of model parameters while only rando variables are treated as fixed at observed values. For the empty model M2 (see equation (2.3)), the likelihood function is given as;

$$
\begin{aligned}
L\left(\theta_{j}, y_{i j}\right) & \propto \prod_{j}\left(\theta_{j}\right)^{y_{i j}}\left(\theta_{j}\right)^{1-y_{i j}} \\
& \propto \prod_{j}\left(\frac{\exp \left(\beta_{0}+\sum_{h=1}^{k} \beta_{h} X_{h i j}+u_{0 j}\right)}{1+\exp \left(\beta_{0}+\sum_{h=1}^{k} \beta_{h} X_{h i j}+u_{0 j}\right)}\right)^{y_{i j}}\left(\frac{1}{1+\exp \left(\beta_{0}+\sum_{h=1}^{k} \beta_{h} X_{h i j}+u_{0 j}\right)}\right)^{1-y_{i j}}
\end{aligned}
$$

which is similarly the classical approach of estimating the unknown parameters $\beta_{0}$ and $\sigma_{u 0}^{2}$.

\subsubsection{Choice of Prior Distribution for Unknown Parameters $\beta$ and $\sigma_{u 0}^{2}$}

Let $\boldsymbol{\beta}=\left(\beta_{0}, \beta_{1}, \ldots, \beta_{k}\right)$ be a vector of fixed effects in equation (2.3). To update the likelihood in equation (2.5) and carry out Bayesian inference on the observed data, the study provided model parameters $\boldsymbol{\beta}$ and $\sigma_{u 0}^{2}$ with suitable prior distributions. For Hierarchical models with smaller number of groups $\mathrm{J} \leq 5$, data is only able to supply little information about $\sigma_{u 0}^{2}$ hence use of non-informative priors $P\left(\sigma_{u 0}^{2}\right)$ may lead to improper posterior distribution thus misleading results [1]. Prior information for multilevel empty model and random intercept models should be in a position to regularize variance of the random effects, $\sigma_{u 0}^{2}$ so as to reduce spread of intercepts among subjects and use of weakly informative priors are highly recommended in the absence of informative priors [5]. Also, for $\mathrm{J} \leq 5$ in the level-2 grouping variable, sampling of the posterior estimates from the posterior distribution using Gibbs sampler in OpenBUGs/ WinBUGs produce reliable results if we have at least 30 observations in each group $j$ and when weakly informative Cauchy prior information, Cauchy $(0,2.5)$ for the level-2 variance $\sigma_{u 0}^{2}$ is used in the absence of informative prior information [5]. In this study we are dealing with a two-level Bayesian Hierarchical approach to model risk of miscarriage. Since reviewed epidemiological studies on modeling risk of miscarriage for subsequent pregnancy outcome have not indicated prior information about the population average of the transformed probabilities $\beta_{0}$ and level-2 variance $\sigma_{u 0}^{2}$, this study resorted to use of weakly informative priors as default prior distributions for both population variance and for population transformed probabilities $\beta_{0}$ [11]. In random intercept models, there is need to reduce the spread of intercepts among-subjects by choosing priors that regularize $\sigma_{u 0}^{2}$ [5]; to achieve this, the study used weakly informative prior distribution $P\left(\sigma_{u 0}\right)$ on the $\sigma_{u 0}$ where $P\left(\sigma_{u 0}\right)$ is assumed to follow a standard Cauchy distribution with $s=0$ and $v=1$ respectively;

$$
\begin{aligned}
& \sigma_{u 0} \sim \text { Cauchy }(s, v) \epsilon[0, \infty] \text { such that } \\
& P\left(\sigma_{u 0}\right) \propto \frac{\Gamma\left(\frac{v+1}{2}\right)}{\Gamma\left(\frac{v+1}{2}\right) \sqrt{v \pi}}\left(1+\frac{\left(\sigma_{u 0}-s\right)^{2}}{v}\right)^{-\left(\frac{v+1}{2}\right)}=\frac{1}{\pi\left(1+\sigma_{u 0}^{2}\right)} ; \text { for all } s=0, v=1,
\end{aligned}
$$

where $s$ is the location parameter and $v$ is the scale parameter. The study settled on standard Cauchy distribution as prior distribution for random effects since the Cauchy prior distribution is a weakly informative prior distribution that yields proper posteriors across all sample sizes, and [13] suggests that $v=1, s=0$ gives a standard Cauchy distribution. To evaluate the performance of the weakly informative prior, the study assessed the accuracy of the posterior estimates in terms of relative bias and compared to the posterior estimate of $\sigma_{u 0}$ when noninformative Uniform prior is used, $\sigma_{u 0} \sim U(0,100)$ [5].For $\beta_{h} ; h=0,1$, the $P\left(\beta_{h}\right)$ was set to be a weakly informative prior following an independent and identically normal distribution with mean $\mu_{\beta_{h}}=0$ and variance $\sigma_{\beta_{0}}^{2}=100$ [8]. Therefore;

$$
P\left(\beta_{h}\right) \propto N\left(\mu_{\beta_{h}}, \sigma_{\beta_{h}}^{2}\right) \text {, for all } \mu_{\beta_{h}}=0, \sigma_{\beta_{h}}^{2}=100
$$

We make $P\left(\beta_{0}\right)$ noninformative by ensuring $\sigma_{\beta_{0}}^{2}$ that is as large as possible, say $\sigma_{\beta_{0}}^{2}=1000$ [16]. The study analyzed and evaluated relative biases of the posterior estimates for $\beta$ and $\sigma_{u 0}^{2}$ in order to assess the accuracy of the model in estimating risk of miscarriage during first trimester of subsequent pregnancy.

\subsubsection{Posterior Distribution for Model Parameters}

The joint posterior distribution of unknown parameters $\beta$ and $\sigma_{u 0}$ in random effect model M2is given as;

$$
\begin{aligned}
& P\left(\sigma_{u 0}, \beta_{h} / y_{i j}\right) \propto L\left(\theta_{j}, y_{i j}\right) P\left(\beta_{h}\right) P\left(\sigma_{u 0}\right) \\
& \propto\left[\prod_{j}\left(\frac{\exp \left(\beta_{0}+\sum_{h=1}^{k} \beta_{h} X_{h i j}+u_{0 j}\right)}{1+\exp \left(\beta_{0}+\sum_{h=1}^{k} \beta_{h} X_{h i j}+u_{0 j}\right)}\right)^{y_{i j}}\left(\frac{1}{1+\exp \left(\beta_{0}+\sum_{h=1}^{k} \beta_{h} X_{h i j}+u_{0 j}\right)}\right)^{1-y_{i j}}\right] \cdot \exp \left[\frac{-1}{2}\left(\beta_{h}-\mu_{\beta_{h}}\right)^{2} \sigma_{\beta_{h}}^{-2}\right] \cdot \frac{1}{\pi\left(1+\sigma_{u 0}^{2}\right)}
\end{aligned}
$$

Equation (2.8) forms the study Bayesian two-level random intercept model (say M2). However, it is difficult to obtain inference about each of unknown parameter $\beta$ and $\sigma_{u 0}$ from the posterior distribution $P\left(\sigma_{u 0}, \beta_{h} / y_{i j}\right)$ since the posterior distribution (2.8) is not in closed form [9]. For this study therefore, Markov Chain Monte Carlo simulation, in specific, Gibbs sampler implemented in OpenBUGS statistical software is used to obtain the posterior estimates from $P\left(\sigma_{u 0}, \beta_{h} / y_{i j}\right)$ [27]. According to [49], the random effect posterior distribution $P\left(\sigma_{u 0}\right)$ only depends on random effects $u_{0 j}$ and not fixed effect $\beta_{0}$ nor the response $y_{i j}$ and therefore, full conditional distribution for $\sigma_{u 0}$ in M2is given as;

$$
\begin{aligned}
& P\left(\sigma_{u 0} / \beta_{h}, y_{i j}\right) \propto P\left(\sigma_{u 0}\right) L\left(\theta_{j}, y_{i j}\right) \\
& \propto\left[\prod_{j}\left(\frac{\exp \left(\beta_{0}+\sum_{h=1}^{k} \beta_{h} X_{h i j}+u_{0 j}\right)}{1+\exp \left(\beta_{0}+\sum_{h=1}^{k} \beta_{h} X_{h i j}+u_{0 j}\right)}\right)^{y_{i j}}\left(\frac{1}{1+\exp \left(\beta_{0}+\sum_{h=1}^{k} \beta_{h} X_{h i j}+u_{0 j}\right)}\right)^{1-y_{i j}}\right] \\
& \frac{1}{\pi\left(1+\sigma_{u 0}^{2}\right)}
\end{aligned}
$$


Similarly, full conditional distribution for $\beta_{h}$ is given as;

$$
\propto \exp \left[\frac{-1}{2}\left(\beta_{h}-\mu_{\beta_{h}}\right)^{2} \sigma_{\beta_{h}}^{-2}\right] \prod_{i j}\left(\frac{\exp \left(\beta_{0}+u_{0 j}\right)}{1+\exp \left(\beta_{0}+u_{0 j}\right)}\right)^{y_{i j}}\left(\frac{1}{1+\exp \left(\beta_{0}+u_{0 j}\right)}\right)^{1-y_{i j}}
$$

In this study, MCMC sampling approach, in specific, Gibbs sampler is used to sample the random parameter estimates $\hat{\beta}_{0}$ and $\hat{\sigma}_{u 0}^{2}$ from the full conditional distributions $P\left(\beta_{h} / \sigma_{u 0}^{2}, y_{i j}\right)$ and $P\left(\sigma_{u 0}^{2} / \beta_{h}, y_{i j}\right)$ respectively.

\subsection{Estimation of the Models}

Maximum likelihood and Bayesian approaches were used to estimate the models M0, M1, M2for classical and Bayesian approaches respectively. Later a comparison was made to determine which approach best fit modeling risk of miscarriage for subsequent pregnancy in women.

\subsubsection{Maximum Likelihood Estimation}

Maximum Likelihood Estimation (MLE) is a statistical approach that involves maximizing a likelihood function in order to estimate a parameter of interest for a given probability distribution [30]. In this study, we first obtained the distribution of our response variables $\boldsymbol{y}_{\boldsymbol{j}}=\left(y_{1 j}, y_{2 j}, \ldots, y_{n_{j} j}\right)$ in cluster $j$ conditional on the random cluster effect $u_{0 j}$, that is;

$$
f\left(\boldsymbol{y}_{\boldsymbol{j}} / u_{0 j} ; \boldsymbol{\beta}\right)=\prod_{j} p\left(y_{i j}=1 \mid u_{0 j}\right)^{y_{i j}}\left(y_{i j}=0 \mid u_{0 j}\right)^{1-y_{i j}}
$$

(see equation (2.5)). The probability of $\boldsymbol{y}_{j}$ conditional on $u_{0 j}$, is as a result of the $j^{\text {th }}$ previous pregnancy outcome cluster observations $\left\{y_{i j}\right\}$ assuming independence given $u_{0 j}$. Suppose $g\left(u_{0 j} ; \boldsymbol{\varphi}\right)$ is the probability distribution of the random intercept $u_{0 j}$ with $\varphi$ as corresponding vector of parameters, and in this study, it is assumed that $u_{0 j} \sim N\left(0, \sigma_{u 0}^{2}\right)$. To fit our two-level model and obtain maximum likelihood estimate for the random effect, the study first obtained unconditional distribution of our response variables $\boldsymbol{y}_{\boldsymbol{j}}$ by multiplying the conditional distribution function with the distribution of the random effects then integrating against the random effect [30]. Therefore, the marginal likelihood function for the $j^{t h}$ previous pregnancy outcome cluster is given as;

$$
L_{j}\left(\boldsymbol{\beta}, u_{0 j}\right)=\int f\left(\boldsymbol{y}_{j} / u_{0 j} ; \boldsymbol{\beta}\right) g\left(u_{0 j} ; \boldsymbol{\varphi}\right) d u_{0 j}
$$

The full marginal likelihood from all the $J$ previous pregnancy outcome clusters is given as;

$$
L=\prod_{j=1}^{J} L_{j}\left(\boldsymbol{\beta}, u_{0 j}\right)
$$

However, the unconditional density (2.13) does not have closed forms thus maximum likelihood estimation resorts to numerical maximization procedure, in specific for this study, Adoptive Guass-Hemite Quadrature estimation method implemented in R software package lme4 is used [18]. Unlike other numerical maximization procedures, Adoptive GuassHemite Quadrature is best suit for this study as since it does not produce biased estimators when fewer number of previous pregnancy outcome clusters $(U=3)$ are used [18].

\subsubsection{Bayesian Estimation}

Unlike the maximum likelihood estimation approach which involve numerical integration to obtain estimates of the interested model parameters, Bayesian inference avoid numerical integration by using Markov Chain Monte Carlo, for our case in specific, Gibbs sampling to repeatedly sample parameter estimates from the respective posterior distribution [8]. In this study, we employ this Bayesian framework by treating our model parameters $\beta_{0}, \beta_{1}, \ldots, \beta_{k}, \sigma_{u 0}^{2}$ and study observations $y_{i j}$ as random variables. Then the study basic models (that is, multilevel empty model M1 (2.2) and random effect model M2 (2.11)) are augmented by assigning fixed effects $\beta=\left(\beta_{0}, \beta_{1}, \ldots, \beta_{k}\right)$ prior distributions $P(\beta)$ and also assigning random effect $u_{0 j}$ a prior distribution $P\left(\sigma_{u 0}^{2}\right)$. In this study, we work with the precisions $\tau=\left(1 / \sigma_{u 0}^{2}\right)$ and not variance $\sigma_{u 0}^{2}$ since our interest is in how much our variable is focused around the mean (that is, how 'precise' our variable is), rather than how 'unprecise' or diffuse it is [8]. Using the Gibbs sampler implemented in the BUGs software package, we estimate the model unknown parameters $\beta_{0}, \beta_{1}, \ldots, \beta_{k}, \sigma_{u 0}^{2}$. The Gibbs sampler samples the parameters from the full conditional distributions; for model M1, the unknown parameters of interest are $\beta_{0}$ and $\sigma_{u 0}$, and therefore Gibbs sampler draws observations in turns from the full conditional distributions $P\left(\beta_{0} / \sigma_{u 0}, y_{i j}\right)$ in (2.10) and $P\left(\sigma_{u 0} / \beta_{0}, y_{i j}\right)$ in (2.9) respectively. For model $\mathrm{M}_{2}$, the unknown parameters of interest are $\beta=\beta_{0}, \beta_{1}, \ldots, \beta_{k}$ and $\sigma_{u 0}^{2}$, and therefore Gibbs sampler draws observations in turns from the full conditional distributions $P\left(\beta_{h} / \sigma_{u 0}, y_{i j}\right)$ in (2.10) and $P\left(\sigma_{u 0} / \beta_{h}, y_{i j}\right)$ in (2.9) respectively. On drawing the samples iteratively, the samples converge to desired joint posterior distributions as number of samples approaches infinity.

\subsection{Model Evaluation and Comparison}

\subsubsection{Intraclass Correlation}

The study evaluates the decomposition of observed variance and between-group variance proportion to aid in model choice between single level and multilevel logistic regression when modeling risk of miscarriage for subsequent pregnancy [16]. The within group variation is assumed to follow a standard logistic regression thus estimated at $\pi^{2} / 3$ [43]. Therefore, the Intraclass correlation coefficient (ICC) is given as; 


$$
I C C=\frac{\widehat{\sigma}_{u 0}^{2}}{\left(\widehat{\sigma}_{u 0}^{2}+\left(\pi^{2} / 3\right)\right)}
$$

In [16], $0 \leq I C C \leq 1$ where $I C C=1$ indicates perfect residual interdependence (that is, within cluster variation does not exist) thus giving us every reason for multilevel modelling. ICC $=0$ indicates perfect residual dependence (that is, between cluster variation does not exist) thus we do not have any reason for multilevel modelling. Though there is no standardized minimum threshold of ICC for multilevel modelling, a coefficient of 0.05 is suggested for multilevel modelling [21].

\subsubsection{MCMC Convergence}

Assessing convergence of MCMC algorithm is essential for the researcher to be assured of reliability of the results before obtaining parameter estimates from the posterior estimates. Autocorrelation and Gelman-Rubin convergence diagnostic plots are used in this study to assess convergence of the chains [8]. Autocorrelation is a situation where sequential draws of interested parameter, for our case $\boldsymbol{\beta}$ and $\sigma_{u 0}^{2}$, from a conditional distribution are correlated leading to a pattern of serial correlation in the Markov chain [22]. Typically, autocorrelation level declines with increasing number of lags in the chain thus indicating convergence of the chains, otherwise, it indicates non-convergence. The Gelman-Rubin convergence diagnostic [15] is applicable in scenarios that involves simultaneous generation of multiple chains. The test involves computing between sample variability $(B S)$ and within sample variability $(W S)$ then comparing the two. Suppose $T$ is the number of iterations for individual chain, then $\frac{B S}{T^{\prime}}$ is the between- sample variance (that is, variance of posterior mean generated over all samples). The foregoing allows us to obtain pooled posterior variance estimate $(\widehat{V})$ using [15]:

$$
\widehat{V}=\frac{T^{\prime}-1}{T^{\prime}} W S+\frac{B S}{T^{\prime}} \frac{g-1}{g}
$$

where $g$ is the number of chains generated. From equation (2.15) we derive the Gelman-Rubin convergence statistic $(\hat{R})$ by dividing equation (2.15) by within sample variability (WS) to get;

$$
\hat{R}=\frac{\widehat{V}}{W S}=\frac{T^{\prime}-1}{T^{\prime}}+\frac{\left(\frac{B S}{T^{\prime}}\right)}{W S} \frac{g-1}{g}
$$

According to [15], if the value of $\hat{R} \rightarrow 1$ then there is a convergence of the model parameter to the target posterior distribution.

\subsubsection{Relative Bias of the Parameter Estimates}

We perform parameter recovery of the two-level model through simulation with cluster bias chosen as the final measurement model. In our proposed hierarchical model, [43]. The relative bias in this case implies the accuracy of the model parameter estimates. Suppose $\omega$ is the true value of our parameter of interest, for which in this study, we set parameter estimates obtained from the real data analysis as the true parameters (that is, for the purpose of this study, $\omega=\left\{\boldsymbol{\beta}, \sigma_{u 0}^{2}\right\}$ ) and $\widehat{\omega}_{r}$ is the posterior parameter estimate of the $r^{t h} ; r=1,2, \ldots, R$ replication. Then we define Bias for the model parameter $\omega$ as;

$$
\operatorname{Bias}_{\omega}=\frac{\sum_{r}^{R}\left(\widehat{\omega}_{r}-\omega\right)}{R}
$$

From equation (2.17), the Bias $_{\omega}$ or the $\mathrm{R}=25$ replications were averaged to provide summary of index called Relative bias is calculated as follows;

$$
\text { Relative } \text { bias }_{\omega}=\frac{\sum_{r}^{R}\left(\widehat{\omega}_{r}-\omega\right) / \omega}{R}
$$

A Relative bias of utmost $|0.2|$ is considered acceptable to conclude that the parameter estimates were accurate, otherwise considered unacceptable [3].

\subsubsection{Model Comparison}

this is essential in examining and selecting a suitable statistical method for modeling the risk of miscarriage among possible models. In this study, we compared the classical methods (that is, the single level logistic model, two-level empty model, two-level random intercept model) and the proposed Bayesian two-level random intercept model in order to determine which among the four approaches is a good fit in modeling risk of miscarriage for subsequent pregnancy among women. AIC and DIC are evaluation measures used in the analysis and comparison in goodness of fit of the models [21][20]. Suppose $d$ is the total number of the number of estimated model parameters, $\varphi$ are the parameters and suppose $\widehat{L}$ is the maximum value of the model likelihood function such that $\hat{L}=\log \left[P\left(y \mid \boldsymbol{\varphi}_{\max }\right)\right]$, then according to [1], the AIC is given as;

and the deviance is also defined as:

$$
\left.A I C=-2\left(\log \left[P{\widehat{\left(y \mid \boldsymbol{\varphi}_{\max }\right.}}\right)\right]-d\right)
$$

$$
D(\theta)=-2 \log (P(\boldsymbol{y} \mid \boldsymbol{\varphi}))+C
$$

where $\boldsymbol{\varphi}$ are unknown model parameters (that is, in this study, $\left.\boldsymbol{\varphi}=\boldsymbol{\beta}, \sigma_{u 0}^{2}\right), \boldsymbol{y}$ are the observations and $P(\boldsymbol{y} \mid \boldsymbol{\varphi})$ represent the likelihood function. $C$ is a constant which does not need to be known since it cancels out for all calculations that compare different models. Suppose $p_{D}$ be effective number of parameters, then $p_{D}$ is the difference between deviance posterior mean $\overline{D(\boldsymbol{\varphi})}$ and deviance evaluated at posterior mean of parameters $D(\overline{\boldsymbol{\varphi}})$, that is;

then, DIC is given as;

$$
p_{D}=\overline{D(\boldsymbol{\varphi})}-D(\overline{\boldsymbol{\varphi}})
$$

$$
\mathrm{DIC}=D(\boldsymbol{\varphi})+2 p_{D}
$$


To determine the model with best fit, the study evaluated the AIC and DIC values and the model with the least value among the AIC and DIC was selected to be the best fit to the data [21].

\section{Results}

\subsection{Assessment of the Multi-Level Structure in Modeling Risk of Miscarriage}

The Bayesian two-level null logistic regression was evaluated to assess the significance of the hierarchical structure in subsequent pregnancy outcome with respect to previous pregnancy outcome. MCMC simulation, in specific, Gibbs sampler using OpenBUGs software was used in three different Markov chains with a total of 15,000 iterations and a thinning of 20 was introduced to control for autocorrelation. A burn-in of 10,000 was discarded and a total of 187,000 samples generated from the full posterior distribution. Weakly informative standard Cauchy prior distribution with mean 0 and scale 1 for the random effects $\left(\hat{\sigma}_{u 0}^{2}\right)$ was used in sampling posterior estimates from the posterior distribution. Convergence of the Markov chains was assessed using autocorrelation plots and Gelman-Rubin Statistics plots. The findings are as shown figures 4.2 and 4.3 and table 4.3 .

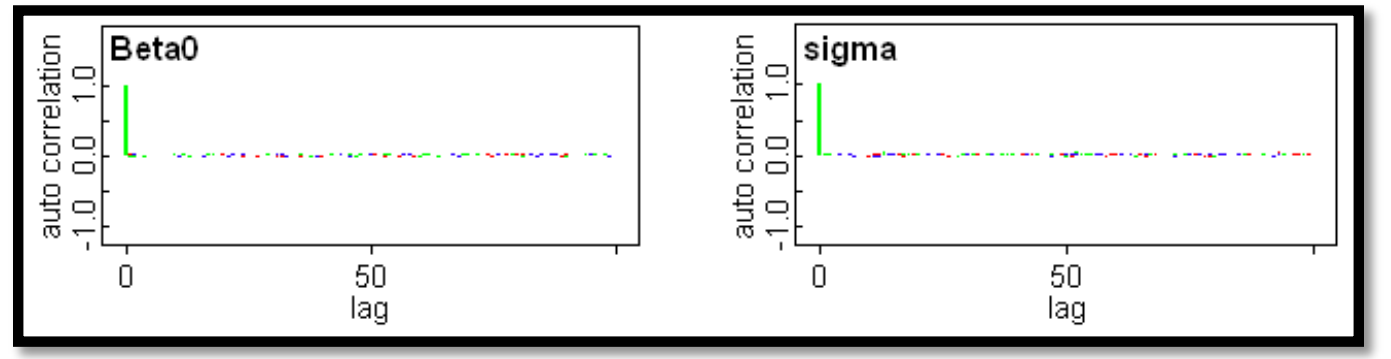

Figure 1: Autocorrelation Plots for Bayesian Empty Model M1

From the autocorrelation plots for each of the regression coefficient (see Figure 2), it is evident that the autocorrelation reduced sharply as the lag increases thus indicating negligible autocorrelation between Markov chains sequences. The three independent chains overlapped with each other showing a good 'chain mixture'; this indicates convergence to the target posterior distribution of the simulated parameter values $\left(\beta_{0}, \sigma_{\mathrm{u} 0}\right)$.

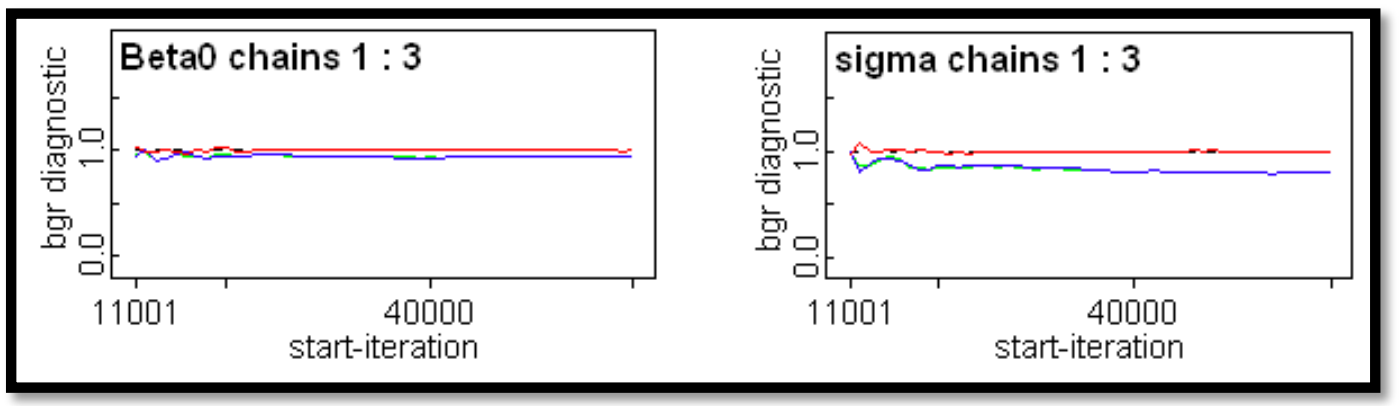

Figure 2: Gelman-Rubin Diagnostic Plots for Bayesian Empty Model M1

In Figure 3, the within group (that is, previous pregnancy outcome clusters) variability is represented by the blue line, the between group variance is represented by the green line. The ratio of between group variance to within group variance is represented by the red line. For all the three regression coefficients, the red line seems exactly on 1 (see Figure 1 ) indicating that the ratio $\hat{R} \rightarrow 1$; this is evident that all the two model parameters $\left(\beta_{0}, \sigma_{\mathrm{u} 0}\right)$ have converged to the target posterior distributions.

\begin{tabular}{|c|c|c|c|c|c|c|}
\hline $\begin{array}{c}\text { Parameter } \\
\text { Estimator }\end{array}$ & $\begin{array}{c}\text { Mean } \\
\text { Estimate }\end{array}$ & $\begin{array}{c}\text { Std. } \\
\text { Deviation }\end{array}$ & MC Error & $\begin{array}{c}\text { Median } \\
\text { Estimate }\end{array}$ & \multicolumn{2}{|c|}{$\begin{array}{c}\text { 95\% Credible } \\
\text { Interval (CI) }\end{array}$} \\
\hline Beta0 $\left(\beta_{0}\right)$ & -0.7309 & 0.7569 & 0.005968 & -0.7218 & -2.271 & 0.7361 \\
\hline Sigma $\left(\sigma_{\mathrm{u} 0}\right)$ & 1.115 & 0.6088 & 0.004737 & 0.9695 & 0.4679 & 2.625 \\
\hline Variance $\left(\sigma_{\mathrm{u} 0}^{2}\right)$ & 0.9399 & & & & & \\
\hline ICC & 0.2222 & & & & & \\
\hline
\end{tabular}

Table 1: Results for the Bayesian Two-Level Empty logistic Model M1

Number of observation $N=115$, groups $\mathrm{J}=3$

The results in Table 3indicates that the overall average log odds of experiencing a miscarriage during first trimester of subsequent intrauterine pregnancy is $\beta_{0}=-0.7309$; this indicates that majority of the pregnant women do not experience miscarriage during first trimester of subsequent pregnancy. The median sigma $\left(\sigma_{\mathrm{u} 0}\right)$ estimated at 0.9695 is found to be significant $\left(\sigma_{\mathrm{u} 0}=0.9695,95 \%\right.$ CI $\left.0.4679-2.625\right)$. The variance at the previous pregnancy outcome level is 
estimated at $\sigma_{u 0}^{2}=0.9399$. The intraclass correlation coefficient (ICC) is estimated at 0.2222 , indicating that the $22.22 \%$ of the variation in the chances of experiencing a miscarriage in the subsequent intrauterine pregnancy during first trimester was attributable to the previous pregnancy outcome clustering.

\subsection{Evaluation of Proposed Bayesian Two-Level Random Intercept Logistic Model M2}

The proposed Bayesian Hierarchical random intercept model M2 (see equation 2.8) with weakly informative standard Cauchy prior distribution with mean 0 and scale 1 for the random effects $\left(\hat{\sigma}_{u 0}^{2}\right)$ and $P(\boldsymbol{\beta}) \sim N(0,100)$, was evaluated to determine its accuracy in the modeling risk of miscarriage for subsequent intrauterine pregnancy during first trimester. Markov Chain Monte Carlo (MCMC) simulation, in specific, Gibbs sampler using OpenBUGs software, was used in three different Markov chains with a total of 200,000 iterations to sample from the posterior distribution. A burn-in of 10,000 was discarded and a total of 60,000 samples were generated from the full posterior distribution. Model Markov Chains convergence was assessed using Autocorrelation plots, and Gelman-Rubin Statistics plot as shown in Figure 4 and 4.5 respectively.
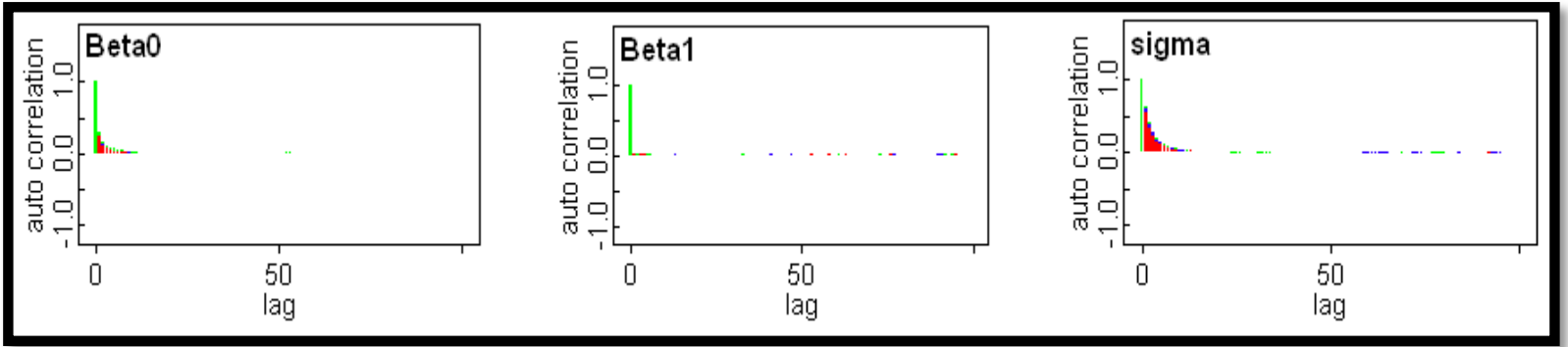

Figure 3: Autocorrelation Plots for the Significant Parameters in the Bayesian Model M2

From the autocorrelation plots for each of the regression coefficient (see Figure 4), it is evident that the autocorrelation reduced sharply as the lag increases thus indicating negligible autocorrelation between Markova chains sequences. The three independent chains overlapped with each other showing a good 'chain mixture'. This indicates convergence to the target posterior distribution of the simulated parameter values $\left(\beta_{0}, \beta_{1}, \sigma_{\mathrm{u} 0}\right)$.

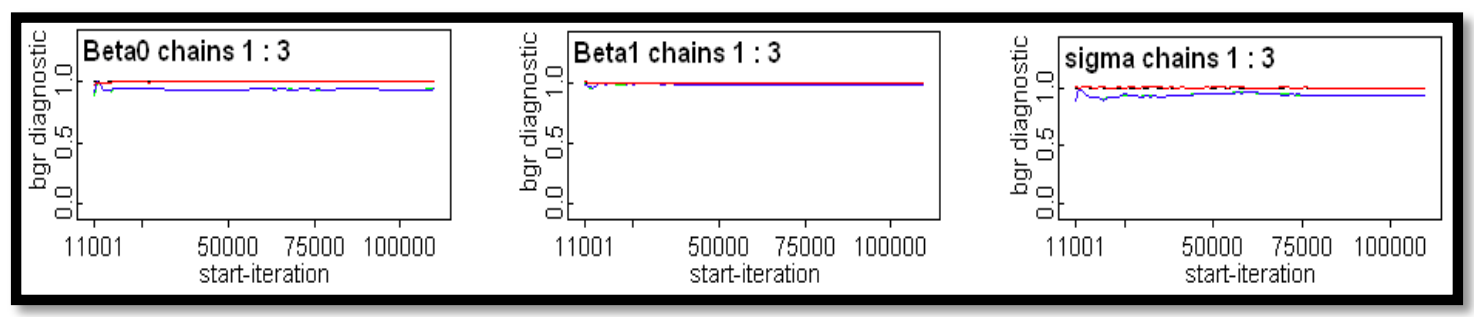

Figure 4: Gelman-Rubin Diagnostic Plots for Significant Parameters in Bayesian Model M2

In figure 4.5, the within group (that is, previous pregnancy outcome clusters) variability is represented by the blue line, the between group variance is represented by the green line. The ratio of between group variance to within group variance is represented by the red line. For all the three regression coefficients, the red line seems exactly on 1 (see Figure 4) indicating that the ratio $\hat{R} \rightarrow 1$; this is evident that all the three model parameters $\left(\beta_{0}, \beta_{1}, \sigma_{\mathrm{u} 0}\right)$ have converged to the target posterior distributions.

\begin{tabular}{|c|c|c|c|c|c|c|c|c|}
\hline Variable & Category & $\begin{array}{c}\text { Mean } \\
\text { Estimate }\end{array}$ & $\begin{array}{l}\text { Std. } \\
\text { dev. }\end{array}$ & $\begin{array}{l}\text { MC. } \\
\text { Error }\end{array}$ & Median & \multicolumn{2}{|c|}{$\begin{array}{l}\text { 95\% Credible } \\
\text { Interval (CI) }\end{array}$} & $\begin{array}{l}\text { Relative } \\
\text { Bias }\end{array}$ \\
\hline \multicolumn{2}{|c|}{ Fixed Part } & & & & & LCI & UCI & \\
\hline Intercept $\left(\beta_{0}\right)$ & & -1.336 & 0.6252 & 0.002466 & -1.34 & -2.547 & -0.0964 & 0.0085 \\
\hline \multirow{2}{*}{$\begin{array}{c}\text { Malaria } \\
\text { Positivity }\end{array}$} & Negative (ref.) & & & & & & & \\
\hline & Positive $\left(\beta_{1}\right)$ & 1.694 & 0.5167 & 0.001415 & 1.691 & 0.6943 & 2.722 & 0.00498 \\
\hline \multicolumn{2}{|c|}{ Random part } & & & & & & & \\
\hline Sigma $\left(\sigma_{\mathrm{u} 0}\right)$ & & 0.8189 & 0.4776 & 0.002775 & 0.7181 & 0.242 & 2.0 & 0.15524 \\
\hline Variance $\left(\sigma_{\mathrm{u} 0}^{2}\right)$ & & 0.6706 & & & & & & \\
\hline ICC & & 0.1693 & & & & & & \\
\hline
\end{tabular}

Table 2: Posterior Parameter Estimates for the Proposed Bayesian Hierarchical

Model M2 with Weakly Informative Prior Information Number of Observation N=115, Groups J= 3 M2= Random Intercept Model with Covariates

Statistical Level of Significance at 5\%; Ref = Reference Category; LCI= Lower CI; UCI = Upper CI

The findings of table 4.4 shows that the log odds of experiencing a miscarriage during first trimester of subsequent intrauterine pregnancy for women who are infected with Malaria significantly increased by 1.694 compared to 
those who are Malaria negative $\left(\beta_{1}=1.694,95 \%\right.$ CI $\left.0.691-2.722\right)$. The intraclass correlation coefficient (ICC) in the proposed Bayesian model M2 with weakly informative prior was estimated at 0.1693 , indicating that the $16.93 \%$ of the variation in the chances of experiencing a miscarriage in the subsequent intrauterine pregnancy during first trimester was attributable to the previous pregnancy outcome. The fact that the sigma $\sigma_{\mathrm{u} 0}=0.8189$ is found to be significant $\left(\sigma_{\mathrm{u} 0}=\right.$ $0.8189,95 \%$ CI $0.242-2.0$ ), it is mathematically equivalent that the ICC $=0.1693$ is significant thus hierarchical multilevel modeling was deemed essential in modeling risk of miscarriage in the subsequent intrauterine pregnancy during first trimester [21]. Posterior parameter estimates shows that the relative bias of the intercept $\left(\beta_{0}\right)$, fixed malaria effect $\left(\beta_{1}\right)$ and the sigma $\left(\sigma_{\mathrm{u} 0}\right)$ are $0.0085,0.00498$ and 0.15542 respectively (see table 4.4 ); these relative bias values were all below the maximum threshold of $|0.2|$ thus considered acceptable [3].

\subsection{Comparison of Classical and Bayesian Models in Modeling Risk of Miscarriage}

\begin{tabular}{|c|c|c|c|c|}
\hline Statistical Approach & Indicators & M0 & M1 & M2 \\
\hline Classical approach & AIC & 147.93 & 134.6 & 125.6 \\
\hline $\begin{array}{c}\text { Bayesian approach with Weakly informative prior; } \\
\sigma_{u 0} \sim \text { Cauchy }(0,1) \text { and } \beta_{h} \sim N(0,100)\end{array}$ & DIC & 147.90 & 127.9 & 121.2 \\
\hline
\end{tabular}

Table 3: Results for Assessment and Evaluation of Model Goodness of Fit

Number of Observation $N=115$, Groups J=3. MO = Single Level Completely

Empty Model, M1= Two-Level Empty Model, M2= Random Intercept Model with Covariates

Model evaluation and comparison results in Table 2 shows that among the three models, the Bayesian approach DIC were less than the AIC, that is, for M0; DIC $=147.90<$ AIC $=147.93$, for M1; DIC $=127.9<$ AIC = 134.6, and for M2; DIC $=121.2<$ AIC $=125.6$. This indicates that updating the pregnancy outcome data with weakly informative prior information improves the performance of the model thus resulting to more accurate results. Comparing the Bayesian models, M1 had largest value of DIC = 127.9 compared to model M0, DIC= 147.90 (see Table 2); this indicates that accounting for the twolevel structure with respect to previous pregnancy outcome grouping improved the performance of the model. The proposed Bayesian Hierarchical two-level logistic model M2 was found to have the lowest information criterion value, DIC = 121.2 compared to Bayesian M1 with DIC = 127.9 and all the other models (see Table 2). This indicates that accounting for the two-level structure effect, the effect of status of Malaria as a risk factor and updating the data with relevant prior information actually improves the performance of the model in estimating risk of miscarriage.

\section{Discussion}

The study formulated a Bayesian two-level random intercept logistic model with the focus of improving on the accuracy in the prediction and estimation of the risk of miscarriage during first trimester of the subsequent pregnancy in women.

Assessment of the hierarchical structure in modeling risk of Miscarriage: Assessment of the two-level structure in the subsequent pregnancy outcome with respect to previous pregnancy outcome using Bayesian two-level null logistic regression M1 shows that intraclass correlation coefficient (ICC) of 0.2222 . The fact that the corresponding $\sigma_{\mathrm{u} 0}=0.9695$ is found to be significant $\left(\sigma_{\mathrm{u} 0}=0.9695,95 \%\right.$ CI $\left.0.4679-2.625\right)$, it is mathematically equivalent that the ICC $=0.2222$ is significant thus hierarchical multilevel modeling is deemed essential in modeling risk of miscarriage in the subsequent intrauterine pregnancy during first trimester [21]. The study findings complement the findings by [9] in a study done in Bangladesh, India who also found out that relative risk of miscarriage in subsequent pregnancy was high among women who had experienced a miscarriage in the previous pregnancy outcome. Our study therefore affirms that there is a hierarchical structure in the subsequent pregnancy outcome with respect to the previous pregnancy outcome clustering thus hierarchical modeling of the risk of miscarriage during first trimester of subsequent pregnancy is essential for reliable results.

Evaluation of Proposed Bayesian Two-Level Random Intercept Logistic Model M2:The study shows that the log odds of experiencing a miscarriage during first trimester of subsequent intrauterine pregnancy for women who are infected with Malaria significantly increased by 1.694 compared to those who are Malaria negative; this indicates that women who are infected with Malaria during first trimester of subsequent intrauterine pregnancy are at higher risk of experiencing a miscarriage compared to those who are not infected with Malaria during the first trimester of intrauterine pregnancy. The findings complement the findings of the study by [12] done in Thailand India which concluded that vivax Malaria was associated with miscarriage during first trimester of pregnancy in women. Comparison of the ICC of $22.22 \%$ for the Bayesian two-level empty model M1 without Malaria risk factor as covariate (see table 4.3) and ICC of 16.93\% for the Bayesian two-level random intercept model M2 with Malaria risk factor as covariate shows a reduction in the variation in the risk of miscarriage attributable to previous pregnancy clustering by $5.29 \%$. The findings complement the study findings by [23] which indicated that the between cluster variations reduced as number of significant covariates were increased in the multilevel model. The posterior estimates for the model M2 parameters $\beta_{0}, \beta_{1}$, and $\sigma_{\mathrm{u} 0}$ were all found to have relative bias below the recommended maximum threshold of $|0.2|$ [3]. This indicates that the estimates were accurate thus would lead to accurate predictions in modelling risk of miscarriage during first trimester of subsequent pregnancy using the proposed Bayesian Hierarchical two-level logistic model M2.

Comparison of Classical and Bayesian Models in Modeling risk of miscarriage: Among the classical and Bayesian models M0, M1 and M2 models, the proposed Bayesian two-level random intercept logistic model M2 is found to have the lowest value of DIC $=121.2$. This indicates that the proposed Bayesian two-level random intercept logistic model M2 is the most plausible model that best fit modeling risk of miscarriage during first trimester of the subsequent pregnancy 
outcome [21]. Based on this finding, the study therefore reveals that accounting for the two-level structure effect, the effect of significant covariates in the model (in the case of this study, covariate is the Malaria positivity as a risk factor) and updating the data with relevant prior information actually improves the performance of the model in estimating risk of miscarriage.

\section{Conclusion}

The main purpose of this research work is to improve the accuracy in modeling risk of miscarriage during first trimester of subsequent intrauterine pregnancy. The study therefore formulated a Bayesian two-level random intercept logistic model M2that takes into account the clustering effect in the subsequent pregnancy outcome, and updates observed data with relevant prior information. The study finds the proposed Bayesian two-level random intercept logistic model M2 to be the most plausible, best fit and more accurate in modeling risk of miscarriage during first trimester of the subsequent pregnancy. It is in this regard that clinicians handling pregnant women in early pregnancy units should use the proposed Bayesian two-level random intercept logistic model M2 in predicting pregnancy outcome during first trimester of a subsequent intrauterine pregnancy. The clinicians should also consider testing Malaria infections and immediately treating women found positive during first trimester of pregnancy to reduce the risk of miscarriage.

\section{References}

i. B. R. Saville, Bayesian multilevel models and medical applications, Journal of Applied Statistics, 16 (2017), no. 3, 210-217, doi.org/10.17615/394k-tf80

ii. Black M, Shetty A, Bhattacharya S. Obstetric outcomes subsequent to intrauterine death in the first pregnancy. BJOG. 115 (2008), no. 2, 269-74. DOI: 10.1111/j.1471-0528.2007. 01562.x

iii. Forero, C. G., \& Maydeu-Olivares, A. Estimation of IRT graded response models: Limited versus full information methods. Psychological Methods, 14 . (2009), no.3, 275-299. DOI: 10.1037/a0015825

iv. G. David Garson, Hierarchical linear modeling: guide and applications. Thousand Oaks, Calif, Sage Publications, New York, 2012. ISBN 978-1-4129-9885-7.

v. Gelman, A. and Hill, J. Data analysis using regression and multilevel/hierarchical models. Cambridge University Press, New York, 2007. ISBN 13: 9780521867061

vi. Andrew Gelman, John B. Carlin, Hal S. Stern, David B. Dunson, Aki Vehtari, Donald B. Rubin, Bayesian data analysis, 3rd Edn, Chapman and Hall/CRC, New York, 2014. ISBN 13: 9781439840955

vii. George I., Argyro S., Nerea M., Jackie A.R. and Kypros H.N. Ultrasonographic Prediction of Early Miscarriage. Human Reproduction, 26 (2011) no.7, 1685-1692. DOI: 10.1093/humrep/der130

viii. Germân R. and Noreen G. Improved estimation procedures for multilevel models with binary response: a casestudy. J. R. Statist. Soc. A,164 (2000), no. 2, 339-355, DOI: 10.1111/1467-985x.00206

ix. H. Hathout, R. Kasrawi, M.A.A. Moussa and A.K. Saleh. Influence of Pregnancy Outcome on Subsequent Pregnancy. International Journal of Gynecology \& Obstetrics, 20 (2012), 145-147, DOI: 10.1016/0020-7292(82)90028-5

x. Jennifer O, Ishwari C, Anna, Samuel M, JeanY, Shannon R, George C. A prediction model for viability at the end of the first trimester after a single early pregnancy evaluation. Australian and New Zealand Journal of Obstetrics and Gynaecology. 53(2013): 51-57, DOI: 10.1111/ajo.12046

xi. Lemoine, N. P. 2019. Moving beyond non-informative priors: why and how to choose weakly 2 informative priors in Bayesian analyses. Oikos117 (2019), 430-438doi: 10.1111/oik.05985

xii. McGready R, Lee SJ, Wiladphaingern J, Ashley EA, Rijken MJ, Boel M et al. Adverse effects of falciparum and vivax malaria and the safety of antimalarial treatment in early pregnancy: a population-based study. The Lancet infectious diseases. 2012;12(5):388-396, DOI: 10.1016/s1473-3099(11)70339-5

xiii. McElreath, R. Statistical Rethinking: a Bayesian course with examples in R and Stan. Taylor \& Francis Ltd. New York, 2020. ISBN: 036713991X

xiv. Model A. New model to predict viability at the end of the first trimester for women with an intrauterine pregnancy of uncertain viability (IPUVI). Ultrasound Obsterics and Gynaecology, 38 (2011): 56-167, DOI: 10.1002/uog.9275.

xv. Ntzoufras I. Bayesian Modeling Using WinBUGS. John Wiley \& Sons Inc. Hoboken, New Jersy, 2009. ISBN: 047014114X

xvi. Raykov T. (2011). Intra-class correlation coefficients in hierarchical designs: Evaluation using latent variable modeling. Structural Equation Modeling: A Multidisciplinary Journal, 18: (2011), no. 1, 73-90, DOI: 10.1080/10705511.2011.534319

xvii. Regan L, Rai R. Epidemiology and the medical causes of miscarriage. Baillieres Best ispepiPract Res ClinObstet Gynaecol. 14 (2000), no.5, 839-854. DOI: 10.1053/beog.2000.0123

xviii. Rodriguez G, Goldman N. An assessment of estimation procedures for multilevel models with binary responses. J. R. Statist. Soc. A. 158 (1995), 73-89, DOI: 10.2307/2983404

xix. Rubio C, Pehlivan T, Rodrigo L, Simon C, Remohi J, Pellicer A. Embryo aneuploidy screening for unexplained recurrent miscarriage: a minireview. Am J Reprod Immunol53 (2005),159-65, DOI: 10.1111/j.16000897.2005.00260.x

xx. Schwarz, G. Estimating the dimension of a model. The Annals of Statistics, 6 (1978), no.2, 461-464. DOI: $10.2307 / 2958889$

xxi. Spiegelhalter, David J.; Best, Nicola G.; Carlin, Bradley P.; van der Linde, Angelika. Bayesian measures of model complexity and fit (with discussion). Journal of the Royal Statistical Society, Series B. 64 (2002), no. 4, 582-639. DOI: 10.1111/1467-9868.00353 
xxii. Train K.E. Discrete choice Methods with Simulation, 2nd Edit. Cambridge University Press, New York, 2009. ISBN: 978-0-521-74738-7.

xxiii. Wu, S., Crespi, C. M., \&Wong, W. K. (2012). Comparison of methods for estimating the intraclass correlation coefficient for binary responses in cancer prevention cluster randomized trials. Contemporary Clinical Trials. 33 (2012), no. 5, 869-880. DOI: https://doi.org/10.1016/j. cct.2012.05.004 